\title{
Difficult intubation and outcomes in intubated patients admitted to intensive care unit
}

\author{
Mohammad Amin Valizade Hasanloei', Ebrahim Hassani', Samira Jahangard ${ }^{r}$, Mehri Piran ${ }^{r}$, Rahimeh Alizadeh Osalou ${ }^{\Delta}$
}

Received 19 Dec, 2016, accepted for publication 19 Feb, 2017

\begin{abstract}
Background \& Aims: Endotracheal intubation is commonly performed in the intensive care unit. Many international guidelines for difficult intubation management are available and the intensivists think that any endotracheal intubation in ICU is potentially difficult with several complications.

Materials \& Methods: After obtaining ethical approval, this descriptive and analytical study was conducted from 2014 to 2015 in the Urmia Emam hospital GICU. Demographic characteristic (Age, Sex), underlying diseases, mean of intubation, mechanical ventilation, ICU and hospital length of stay, frequency of difficult intubation, drugs used for intubation, mortality of patients were extracted from the medical information unit and entered to check lists. The data were analyzed with SPSS software ver.20. . P $<0.05$ was considered significant.
\end{abstract}

Results: The mean of variables were as follows: intubation period 14.56 \pm 4.71 , mechanical ventilation $13.24 \pm 5.54$, ICU length of stay $18.11 \pm 10.16$ and hospital $21.04 \pm 13.09$ days. Frequency of difficult intubation was $9.31 \%$. Mean number of attempts was 1.31 times for intubation and frequency of mortality was $35.17 \%$. The Mean of intubation, mechanical ventilation, ICU and hospital length of stay period between 2 groups (difficult and non difficult intubation) were significantly different $(\mathrm{P}<0.05)$, although mortality frequency was not significant.

Conclusion: This study, like previous studies showed that difficult intubation had a negative influence on patients' outcome in the intensive care unit.

Keywords: Difficult Intubation, Outcome, Intensive care unit

Address: Ayatollah Kashani Ave, Patient Safety Research Center, Urmia, Iran

Tel: +989144480050

Email: Ehassani87@gmail.com

\section{Introduction}

Endotracheal intubation in the ICU is a necessary intervention that is frequently performed and compared to the operating room is a different clinical aspects (1).
Airway management is performed frequently in everyday work of anesthesia, but sometimes in outside the operating room is vital. Several studies of airway insertion outside the operating room, have identified high rates of complications,

\footnotetext{
${ }^{1}$ Associate professor, fellowship of intensive care medicine, Urmia University of Medical Sciences.

${ }^{2}$ Associate professor, fellowship of cardiac Anesthesia, Urmia University of Medical Sciences. (corresponding authors)

${ }^{3}$ General practitioner, Urmia University of Medical Sciences.

${ }^{4}$ Nursing, Urmia University of Medical Sciences

${ }^{5}$ Nursing, Urmia University of Medical Sciences
} 
including intubation failure, esophageal intubation, hypoxemia,... that includes studies in the ICU and the emergency (2). Intubation for a period of time for critical ill patients was considered to be potentially unstable and its international guidelines on the management of difficult intubation are available (3). Endotracheal intubation for airway stabilization seems to increase risk of lifethreatening complications such as severe hypoxemia, esophageal intubation, aspiration and increase of cardiac arrest. Clearly, these effects will reduce with the glottis better viewing (4). Improvement and validation studies, provide a recommendation to simplify the scoring system for identifying patients with difficult intubation in the ICU that have 7 sections including Mallampati score 3 and 4, obstructive sleep apnea syndrome, reduced mobility of the cervical spine, limited of mouth opening, pathology result existence (sever hypoxemia, coma) and non-anesthesiologist performed intubation. Based on these factors was provided MACOCHA (5). On the other hand, we know that a comfortable experience of Laryngoscopy does not ensure patient's airway without problems and aging and airway pathology may change airway (6). Taking into account these considerations in the ICU, each endotracheal intubation should be considered potentially difficult intubation and the existing algorithms based on ICU needs to be changed because of difficult endotracheal intubation increases mortality, morbidity and disability in patients of intensive care units (7). Several studies have identified difficult airway insertion. Denmark database of anesthesia offered 4 parameters as risk factors for difficult intubation, including obesity, avoidance of neuromuscular blockers, history of previous difficult intubation, adjusted Mallampati scores. However, obese, Mallampati score and history of previous problems with intubation is not a sufficient test for predicting difficult endotracheal intubation (8).

A study in the UK in 2011 showed that more than $60 \%$ of adverse events associated with airway in ICU led to death or severe brain damage.

The two studies with regard to the complications in patients admitted to intensive care units summarized involving issues such: multi-organ failure, elderly, use of vasopressor and low responsiveness to fluid administration, lack of space and lack of easy access to the patient's head, the emergency of the endotracheal intubation and the number of attempts done, severe hypoxemia, regurgitation and aspiration, bradycardia and cardiac arrest $(9,10)$. The importance of preserving and maintaining airway and challenges facing physicians, working in intensive care units, especially when exposed with difficult intubation and implications which increase mortality and morbidity, we decided to perform this descriptive and analytical study in the General Intensive Care Unit (GICU) of the Urmia Emam hospital.

\section{Materials \& Methods}

This retrospective, descriptive and analytical study, After obtaining ethical approval from Urmia University of Medical Sciences,was conducted from 2014to 2015) to examine the frequency of difficult intubation and outcomes in patients admitted to general intensive care units (GICU) of the Urmia Emam hospital. In this study, the medical records of patients admitted to the intensive care unit who had endotracheal intubation under direct laryngoscopy were studied. Patients also had received medication such as opioids, benzodiazepines, hypnotics during endotracheal intubation. Demographic characteristic (Age, Sex), underlying diseases, mean of intubation, 
mechanical ventilation, ICU and hospital length of stay, frequency of difficult intubation, drugs used for intubation, mortality of patients were extracted from the medical information unit and entered to check the list. The $\mathrm{t}$ test was used for statistical analysis, and $\mathrm{p} \leq 0.05$ was used as an index of statistical significance. In this study difficult intubation was defined as follows:

: 3 or more than 3 attempts should be made at direct laryngoscopy. . All intubations were performed by fellowship of critical care medicine with more 10 years working in ICU. Collected data were analyzed by SPSS software version 20 .

\section{Results}

In this study, records of 580 patients admitted to the general intensive care unit (GICU) during the 2 year period $(2014,2015)$ at Urmia Emam hospital were investigated. The mean age of was 56.67 \pm 26.25 years (minimum 14, maximum 102 years). Of 580 patients 306 (52.75\%) were male and 274 patients $(47.25 \%)$ were female.

Of 580 patients were diagnosed as follow:, 236 (40.69\%) multi-trauma, 134 (23.10\%) pulmonary diseases, 125 cases (21.55\%) CNS, 61 patients $(10.52 \%)$ after surgery, $24 \quad(4.14 \%)$ other cases.(Table 1).

Table 1. show intubation, mechanical ventilation, ICU and Hospital length of stay periods(Days).

\begin{tabular}{llll} 
Max & Min & Mean \pm SD & Variable \\
\hline 38 & 5 & $4.71 \pm 14.56$ & Intubation Period (Day) \\
112 & 6 & $6.5413 .24 \pm$ & Mechanical Ventilation (Day) \\
112 & 6 & $8.4918 .11 \pm$ & ICU Length of stay(Day) \\
112 & 8 & $10.68 \pm 21.04$ & Hospital Length of stay (Day) \\
\hline
\end{tabular}

The frequency of difficult intubation was 54 cases $(9.31 \%)$ and 526 patients $(90.69 \%) \%$ ) had no difficulty in intubation.

Commonly used medications during intubation were midazolam, fentanyl, lidocaine, thiopental.

Frequency of hypoxemia (Spo $2<90 \%$ ) for 44 patients was reported $(7.58 \%)$.
The rate of first, second, third and fourth attempt intubation were 477 (82.2\%), 49 (8.4\%), 31 (5.3\%) and $23(4 \%)$, respectively.

The mean number of attempts was 1.31 times laryngoscopy.

Table 2. show comparison of intubation, mechanical ventilation, ICU and Hospital length of stay between 2 group (With or without difficult intubation). Independent T-Test was used to test to analysis.

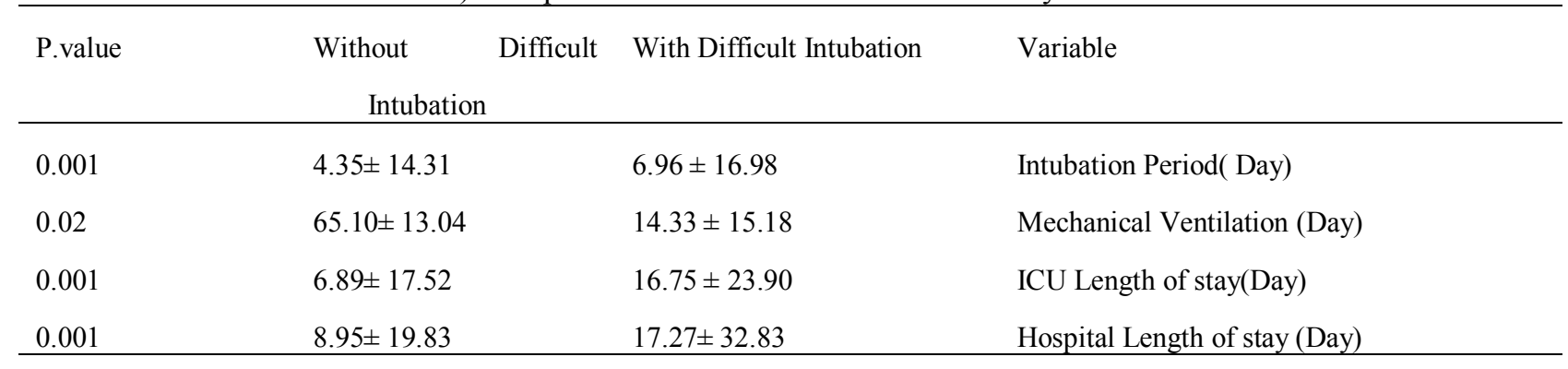


204 patients (35.17\%) died and 376 patients (64.83\%) were discharged .

Of 54 patients with difficult intubation, 9 patients $(16.7 \%)$ were died, and 45 patients $(83.3 \%)$ were discharged with recovery.

Of 526 patients intubated without problems, 331 patients $(62.9 \%)$ were discharged and 195 patients $(37.1 \%)$ were died.

\section{Discussion and conclusion}

The current study was conducted in the general intensive care unit (GICU) in Urmia. In this study, 580 patients were investigated. The mean years of age was $56.67 \pm 26.25$ predominating, by male $(52.75 \%)$. The frequency of difficult intubation was 54 cases (9.31\%) with a mean attempt of laryngoscopy 1.31 times. $35.17 \%$ of the patients were died and the rest were discharged. The average duration of intubation, mechanical ventilation, intensive care unit and hospital length of stay between the two groups with and without difficult intubation had statistically significant difference $(\mathrm{P}<0.05)$.

Joffe A, et al (1) in 2009 commented that the $22 \%$ of patients in their study had difficult intubation that increases risk of tracheostomy, mortality and morbidity while our study showed that there was not any difference between the numbers of days of mechanical ventilation and intensive care unit length of stay among groups. In our study, the frequency of patients with difficult intubation was $9.31 \%$ that was lower than the figures reported by Jofte A, et al (1. With regard to days of intubation, mechanical ventilation and hospitalization in ICU and our study showed there was a significant difference between groups. Unlike Jeffe and his colleagues' study this survey indicated that there was not a significant difference in the mortality of two groups. In fact, the number of low mortality in patients with difficult intubation (9 patient) prevents comparison of the high number of deaths in patients without difficult intubation, this represents that other reasons acts in the mortality of patients beside difficult intubation. On the other hand, our study was conducted during the 2 year period with 580 patients, which also differs our study from Jeffes's.

Cook TM, et al. (2) in 2011 in England reported that 36 patients out of 184 patients in their study had difficult intubation, and $61 \%$ of cases were death or sustained permanent nerve damage. The poor identification of $\mathrm{t}$ patients at risk, poor planning or incomplete and insufficient measurement (human, equipment) to manage problems were introduced by Cook's study as major factors of high death rate. Noppen RR et al. (4) in 2012divided intubated patients in two groups (using a Macintosh laryngoscope and video laryngoscopy C- MAC) and finally concluded that it seems video laryngoscopy in the intensive care unit (in case of potentially difficult intubation) is a useful tool, because it provides a good view to incubate. Our study as Noppens and his colleagues' (4) study was performed during the 2-year period, but taking into account the number of patients, our samples were double compared with Noppens and his colleagues' study. , also we only used simple laryngoscope in our study. Unlike Cook TM, et al.' study (2) approximately $20 \%$ of patients have had difficult intubation in their study and half of them $(9.31 \%)$ had difficult intubation and poor outcomes which is more than our study may be due to the factors related to the severity of diseases and organ failure.

Heuer JF et al. (7) in 2012 commented that difficulty in endotracheal intubation increase mortality and morbidity and suggested that educational programs on the alternative methods of airway management in cases of difficult intubation should be presented. 
Also, $23 \%$ of their patients had difficult intubations that were higher compared to our study. They also commented that the difficult intubation is associated with difficult bag-mask ventilation, oxygenation problem and anatomical anomalies. Lundsteorn LH, et al (8) in Denmark in 2012 commented that four variables such as obesity, history of difficult intubation and modified Mallampati score and avoidance of neuromuscular blockers are associated with difficult intubation but none alone is not a enough predicting factor for difficult intubation.. We didn't access to the previous history of our patient's intubation difficulties and also for the majority of patients in intensive care units the evaluation of Mallampati and airway class has been always not possible. Also the policy of our intensive care unit due to the numerous problems is avoiding the utilization of neuromuscular blockers and despite these limitations, it seems, difficult intubations in our case-study were lower than similar studies.

Noppens RR (4) in 2012 commented that the alternative equipments such as laryngeal mask airway, fiber optic and MC Coy laryngoscope in cases with difficult airway are not normally present. In cases of difficult intubation, we only augmented time of attempts of laryngoscopy and used MC Coy blades and endotracheal tube style and the fiberoptic weren't used due to the lack of easy access in case of emergency. The accessibility to laryngeal mask airway and MC Coy laryngoscope in our study was easier than Noppers study (4), Lavery GG and his colleagues (11) in 2009 commented that all patients admitted to the intensive care unit should be considered as difficult intubation potentially, and ICU physicians are exposed to potential airway problems with insertion and removal of the tube, so must be expert in the difficult airway algorithm. Our study was done during 2 years with appropriate sample size, which showed about $10 \%$ of patients who were admitted to the intensive care unit had difficult endotracheal intubation. This may be influenced the outcome of patients specially the increase mean duration of ICU, hospital length of stay, mechanical ventilation and intubation period but there was no difference in mortality between the two groups. Such outcomes may be explained by the involving factors such as the severity of the underlying diseases and organ failure in patients admitted to the intensive care unit.

We do not know the reason for the low number of cases of difficult intubation for the patients admitted to our intensive care unit was personal characteristics of patients, such as less involvement of the anatomy of the airway, the physical phenotype of patients, or expertise of persons working on this ward, or a combination of these factors. In our study, we did not use for patients the neuro-musculer relaxants, and the dose of medication used for sedation and intubation was less than the recommended dose for induction anesthesia which consist one of our limitations. This matter is in contrary to the Joffe A, et al (1) and Lundsteom LH, et al (8) studies where the role of neuromuscular blockers to reduce the risk of difficult intubation was emphasized. It seems that in case of using a sufficient dose of sedative drugs in our study, we would encounter with a fewer rate of difficult intubation.

Finally, our study, like previous studies showed that difficult intubation for patients admitted to the intensive care unit happens and there are carry high risks for patients.

Such complications were seen in our study and were similar to previous studies.

With regard to the rate of mortality increase, our study in contrast to previous studies, there was no significant difference between the two studied groups. 
It is recommended that physicians working in intensive care units be aware of difficult airway algorithm and periodic training programs and workshop should take place. In addition, the replacement of equipments in the intensive care unit should be considered on a regular basis. Finally, it is recommended to implement a comprehensive study with more patients to obtain better outcomes and to see less cases of difficult intubation in the intensive care unit.

\section{References}

1- Joffe A, Liew E, Matioc A, Willmann K. Unanticipated difficult intubation in the medicalsurgical intensive care unit: its effect on outcome of critically-ill adults. Internet J Anesth 2009; 24(1):1-6.

2- Cook TM, Woodall N, Harper J, Benger J; Major complications of airway management in the UK: results of the Fourth National Audit Project of the Royal College of Anaesthetists and the Difficult Airway Society. Part 2: intensive care and emergency departments. Br J Anaesth 2011; 1-11.

3- McGrath B, O’Donohoe E, Waldmann C. Airway Management in Critical Care-New Guidelines, Old Problems [Internet]. SAGE Publications; 2012 [cited 2017 Mar 1]. Available from: http://inc.sagepub.com/content/13/2/100.full.pdf
4- Noppens RR, Geimer S, Eisel N, David M, Piepho T. Endotracheal intubation using the C-MAC ${ }^{\circledR}$ video laryngoscope or the Macintosh laryngoscope: a prospective, comparative study in the ICU. Crit Car 2012;16(3):R103.

5- Kritek P. Predicting difficult intubation- easier to say who isn't at risk. J Watch Jeneral Med 2013; 1-5.

6- Hagberg CA, Artime CA. Airway management in the adult. In: Miller RD, Cohen WH, Eriksson LT, Flisher LA, Wiener - Kronish JP, Young WL. Millers anesthesia. 8th ed. Elsevier; 2015. P.1647-83.

7- Heuer JF, Barwing TA, Barwing J, Russo SG, Bleckmann E, QuintelM, et al. Incidence of difficult intubation in intensive care patients: analysis of contributing factors. Anaesthesia and Intensive Care 2012; 40(1):120-7.

8- Lundstrøm LH .Detection of risk factors for difficult tracheal intubation. Danish Med J 2012, 59(4):1-20.

9- Jaber S, AmrAoui J, Lefrant JY, Ari CH C. Clinical practice and risk factors for immediate complications of endotracheal intubation in the intensive care unit: a prospective, multiple-center study. Crit Care Med 2006;34: 2355-61.

10- Rüdiger R, Noppens B. Airway management in the intensive ca re unit. Acta Clin Croat 2012; 51:511-7.

11- Lavery GG, McCloskey BV. The difficult airway in adult critical care. Crit Care Med 2009; 36(7): 216373. 